\title{
Evaluación estadística en el número de publicaciones y citaciones en el área de Artes y Humanidades en Latinoamérica, entre 1996-2018
}

\author{
Fernando Restrepo Betancur \\ Universidad de Antioquia, Medellín (Colombia). Grupo STATISTICAL \\ Correo-e: frbstatistical@yahoo.es | ORCID iD: http://orcid.org/0000-0002-8583-5028
}

Recibido: 21-05-19; 2a versión: 17-09-19; Aceptado: 15-12-19.

Cómo citar este artículo/Citation: Restrepo Betancur, F. (2020). Evaluación estadística en el número de publicaciones y citaciones en el área de Artes y Humanidades en Latinoamérica, entre 1996-2018. Revista Española de Documentación Científica, 43 (4), e277. https://doi.org/10.3989/redc.2020.4.1688

Resumen: La clasificación de las revistas científicas es de vital interés en la comunidad académica, por lo que se tuvo como objetivo comparar el número de publicaciones y citaciones en Artes y Humanidades en sus distintas áreas del conocimiento, en los países de Latinoamérica, de acuerdo con el reporte efectuado por Scimago Journal and Country Rank, entre los años 1996 a 2018. En el estudio se emplearon las técnicas estadísticas: análisis de clúster, componentes principales y MANOVA. Como resultado, se destacan diferencias entre países en lo relacionado con el número de publicaciones y citaciones, donde Brasil, México, Argentina, Chile y Colombia son los de mayor producción académica, y Surinam, Guyana, Haití, Honduras y Paraguay los de menor producción. Se concluye que existe una gran brecha entre las naciones de Latinoamérica en las estadísticas asociadas con las publicaciones en el área objeto de estudio.

Palabras clave: Artes y Humanidades; artículos científicos; auto-citaciones; citaciones; índice H; Latinoamérica; publicaciones.

\section{Statistical evaluation in the number of publications and citations in the area of Arts and Humanities in Latin America, between 1996-2018}

\begin{abstract}
The classification of scientific journals is of vital interest for the academic community. The aim of this article is to compare the number of publications and citations in Arts and Humanities, in their different areas of knowledge, in Latin American countries according to the report by Scimago Journal and Country Rank, between 1996 and 2018. In the study several statistical techniques were used: cluster analysis, main components and MANOVA. As a result, differences between countries were detected in relation to the number of publications and citations, where Brazil, Mexico, Argentina, Chile and Colombia are the ones with the highest academic production, while Suriname, Guyana, Haiti, Honduras and Paraguay have the lowest. It is concluded that there is a large gap between the nations of Latin America on statistics associated with publications in the area under study.
\end{abstract}

Keywords: Arts and Humanities; scientific articles; self-citations; citations; H index; Latin America; publications.

Copyright: (c) 2020 CSIC. Este es un artículo de acceso abierto distribuido bajo los términos de la licencia de uso y distribución Creative Commons Reconocimiento 4.0 Internacional (CC BY 4.0). 


\section{INTRODUCCIÓN}

Una revista científica constituye el medio de difusión del conocimiento generado por los grupos de investigación, donde se establecen una serie de requisitos a fin de que la comunidad científica pueda comparar resultados. En 1665 se dio a conocer la primera impresión referente a una revista de tipo científico, la cual se denominó "Journal des Sçavans" (López, 2016).

Las revistas científicas sirven de canal de difusión de las diferentes investigaciones que se llevan a cabo por entes públicos o privados. Es el medio que utilizan las comunidades científicas para intercambiar información derivada de sus experiencias experimentales o de trabajo de campo. Estos medios permiten que, a nivel internacional, se conozcan los avances en un área determinada del conocimiento, beneficiando a la sociedad científica en aspectos relacionados con la innovación o la invención de nuevos productos, que conducen a mejorar la calidad de vida. En la actualidad, el prestigio de los investigadores y de los grupos a los cuales están adscritos, es juzgado por la calidad de la información que se plasma en los diferentes artículos derivados de sus procesos investigativos. El reconocimiento se da por las citaciones que otros investigadores realizan en nuevos artículos, asociados con el tema de interés que se trata (Delgado y otros, 2006).

Las revistas científicas se utilizan por parte de la comunidad de investigadores como medio de debate intelectual de los avances en el conocimiento (Rozemblum y otros, 2012). Existen entidades nacionales e internacionales que cualifican el grado de calidad de las revistas científicas que se editan en diversas áreas del conocimiento (Benavent y otros, 2007). Diferentes variables de tipo cualitativo y cuantitativo se tienen en cuenta al momento de evaluar una revista especializada, donde es muy importante la puntualidad oportuna en la publicación (Testa, 2009). Para que una revista científica sea analizada en las bases de datos mundiales, debe cumplir ciertas normas editoriales que permitan tener rigurosidad en el manejo de la información que allí se exprese. Esto con el fin de garantizar que lo que se edita es válido (Delgado y otros, 2006). Dos aspectos importantes se deben tener en cuenta: el factor de impacto de la revista y la frecuencia de citación (Beltrán, 2006).

La clasificación de una revista científica es de vital interés para los entes gubernamentales o privados, con el fin de brindar apoyo económico en futuras investigaciones donde participen los diferentes grupos científicos. Los expertos en aspectos bibliométricos utilizan las bases internacionales de las revistas, con el fin de establecer políticas de es- tandarización, evaluación de la productividad, número de citas recibidas, entre otros aspectos. Las universidades pueden evaluar a los investigadores adscritos a ellas, de acuerdo con el número de publicaciones que se hayan generado en un periodo determinado, y a la vez ellas también son marco de referencia internacional, en la comparación que se haga entre universidades con base en la productividad de los docentes (Torres y otros, 2010).

Gross y Gross, fueron los pioneros en sugerir que el número de citaciones de un artículo científico fuera empleado como indicador de la calidad del mismo. En los estudios bibliométricos, el factor de impacto (indicador que permite evaluar la calidad de una revista, que varía entre las diferentes áreas del conocimiento), constituye uno de los pilares fundamentales en la ayuda de los bibliotecarios, con el fin de enaltecer las revistas más destacadas dentro de cada área del conocimiento (Beltrán, 2006). En España, profesionales relacionados con la documentación científica, empezaron a ponderar las revistas existentes, donde este concepto ha ido evolucionando de manera favorable a través del tiempo. A comienzos del nuevo milenio, en España se solicitó a uno de cada cinco profesores universitarios, clasificar cerca de 900 revistas relacionadas con Ciencias Sociales y Humanas, a fin de obtener la primera clasificación valorativa de estos medios de comunicación científicos (Alcaín y otros, 2008).

El factor de impacto (indicador del número medio de citas recibidas por los artículos de una revista en un período de tiempo), presenta una serie de inconvenientes al momento de valorar una publicación. Uno, asociado al número de citaciones en un periodo de dos a tres años, y otro se asocia a que no importa el medio donde aparece el artículo, otorgando igual ponderación si la revista es de alto prestigio o no, por lo que se han propuesto formas alternativas de cualificación, como el factor de impacto promedio y ponderado (Buela, 2003). El índice $\mathrm{H}$ es un indicador que califica la producción científica, en relación con la cantidad y el número de citas recibidas en cada una de las publicaciones incluidas en la base de Scimago. Considerando que dicho índice es aplicable a investigadores, revistas y naciones que dan origen a las publicaciones, cuanto más alto sea el valor $\mathrm{H}$, mayor impacto presenta la revista. Con el fin de garantizar la idoneidad de un medio escrito científico, se aceptó a nivel internacional, que cada artículo debería ser revisado por pares externos, los cuales expresan el concepto referente a un tema tratado, donde argumentan los pro y contra del artículo, algo que permite, en muchos casos, efectuar ajustes que hacen que la calidad del mismo se incremente (Romanos, 2008). 
Las escuelas de bellas artes, en España, se transforman en facultades de índole universitaria a partir de un Decreto Real, según el cual los docentes han de ser evaluados en su desempeño, a fin de ubicar el sistema educativo acorde con las políticas de la Unión Europea. Lo anterior implica que las revistas en el área de Artes se deben colocar a tono, para tener un posicionamiento científico de alto valor, de donde se hace necesaria una evaluación constante de su calidad (Caerols y otros, 2017).

Esta investigación tiene como objetivo comparar el número de publicaciones en Artes y Humanidades, en sus distintas áreas del conocimiento, en los países de Latinoamérica, de acuerdo con el reporte efectuado por Scimago Journal and Country Rank, entre los años 1996 a 2018. De manera adicional, se evalúa del número de documentos citados, el número total de citaciones, auto-citaciones, citaciones por documento e índice $\mathrm{H}$.

\section{METODOLOGÍA}

Se tomaron los registros de libre acceso reportados por Scimago Journal and Country Rank en Latinoamérica, en Artes y Humanidades, en las áreas $\mathrm{T} 1=$ Arqueología, T2=Artes y Humanidades, T3=Los Clásicos, T4=Conservación, T5= Historia, T6=Historia y Filosofía de la Ciencia, T7=Lenguaje y Lingüística, T8=Literatura y Teoría Literaria, T9=Museología， T10=Música，T11=Filosofía, T12=Estudios Religiosos, T13=Arte Visual y Actuación en Arte, entre los años 1996 a 2018, creando una base de datos en el paquete Excel versión 2010, con las variables: $\mathrm{V} 1=$ número de publicaciones; V2=número de documentos citados; V3=número total de citaciones; V4=auto-citas; V5=citaciones por documento y $\mathrm{V} 6=$ índice $\mathrm{H}$. Como variables control figuran el país y el año.

Para el proceso de la información se aplicaron las técnicas estadísticas: comparación por medio del modelo GLM, que permite detectar diferencias estadísticas entre países y entre áreas del conocimiento, donde se aplicó transformación de datos basado en la familia BOX-COX, a fin de validar los supuestos asociados con el modelo, determinando el valor lambda óptimo para realizar la citada transformación. Se complementó con la prueba de contraste de Tukey, al nivel de significancia de $5 \%$. El análisis se complementó con la prueba multidimensional MANOVA, que permite incorporar todas las variables de interés en las comparaciones efectuadas. Finalmente, se empleó el método de componentes principales para establecer el constructor de los ejes asociados a las áreas temáticas relacionadas con Arte y Humanidades, análisis que permite establecer la importancia de cada variable evaluada. Para el proceso de la información se utilizó el paquete estadístico de libre acceso SAS University.

\section{RESULTADOS Y DISCUSIÓN}

De acuerdo con la Real Academia Española de la Lengua, publicar significa "Difundir por medio de la imprenta o de otro procedimiento cualquiera un escrito", y publicación, la "acción y efecto de publicar". Publicar se hace vital para cualquier persona que trabaje en la ciencia, ya que divulga el conocimiento producido, da a conocer a su grupo y se proyecta como profesional ante la comunidad internacional. Una de las dificultades que se encuentra es que muchos investigadores dejan sus resultados en los anaqueles, por la dificultad que tienen para escribir (Jiménez, 2015). En la presente investigación, se aprecia que el área denominada los clásicos, es la de menor número promedio de publicaciones, y por tanto de citaciones, lo que puede obedecer a que el tema sea más complejo que los demás, presentándose, por ende, menos difusión en las diferentes revistas acreditadas, contrario a lo que sucede con Historia y Filosofía, como se aprecia en la (Tabla I).

La investigación científica, traducida en publicación, es el objetivo que debe tener cualquier persona dedicada al campo científico o a la docencia, con el fin de dar a conocer su conocimiento y permitir, a través de él, la generación de discusiones académicas para bien del progreso en un área específica, de manera tal que se puedan contrastar hipótesis y efectuar reingenierías en futuros procesos investigativos (Bienert y otros, 2015). En Artes y Humanidades, las áreas con mayor número promedio de publicaciones son: T6=Historia y Filosofía de la Ciencia, $\mathrm{T} 2=$ Artes y Humanidades, T5 $=$ Historia y T8=Literatura y Teoría Literaria, y las menores son: T3=Los Clásicos, T4=Conservación, T10=Música, T11=Filosofía, T12=Estudios Religiosos, presentándose diferencias. Similar comportamiento sucede con el número de documentos citados.

Respecto al número total de citaciones, el mayor valor se dio en T7=Lenguaje y Lingüística, el cual se diferencia de los demás temas, sucediendo igual dinámica para las variables V4=auto-citaciones, $\mathrm{V} 5=$ citaciones por documento y $\mathrm{V} 6=$ índice H. El tema T3=Los Clásicos, es el de menor producción académica. El mayor índice $\mathrm{H}$ se dio en T7=Lenguaje y Lingüística, seguido de T2=Artes y Humanidades. Lo anterior evidencia grandes diferencias en el número promedio de publicaciones, en el área de Artes y Humanidades. El análisis multivariado de la varianza MANOVA, que involucra todas las variables evaluadas, permitió establecer diferencias marcadas, como se aprecia en la Tabla I. 
Una crítica que se hace a algunas revistas de difusión científica, se relaciona con la calidad de sus publicaciones, por lo cual se han generado indicadores donde se muestra su verdadero impacto, permitiendo clasificarlas de acuerdo con múltiples factores de relevancia científica (Andronic y otros, 2017). El índice $H$ presenta diferencias estadísticas, donde el área T7=Lenguaje y Lingüística posee mayor valor, con 41, las áreas de menor impacto son: T3=Los Clásicos, T11=Filosofía, T12=Estudios Religiosos, T13=Arte Visual y Actuación en Arte, presentando el índice $\mathrm{H}$ más bajo (Tabla I).

Antes de someter un artículo, los investigadores se fijan en un medio de publicación científica que les garantice mayor calidad en el proceso y brinde mayor factor de impacto, lo que trae como ventaja la posibilidad de contactos entre investigadores, adicionalmente permite acceder a fuentes de recursos, y de igual manera facilita un debate crítico y objetivo entre pares académicos, entre muchas otras ventajas. En el área de Artes y Humanidades, Brasil, México, Chile, Argentina y Colombia, son las naciones donde se publica más, por lo tanto reciben el mayor número de citas, al igual que poseen el mejor valor de índice $\mathrm{H}$. La elección de una revista donde se desee publicar los resultados derivados de un proceso investigativo, depende de múltiples factores, entre los que se destaca el idioma en el cual publica el medio de difusión científica. La mayoría de revistas se emiten en inglés y quienes no tienen suficiente conocimiento de ese idioma, encuentran en ello una dificultad metodológica, y algunos científicos gustan publicar sus artículos en su lengua natal (Acuña y otros, 2013). Surinam es el país con las estadísticas más bajas en relación a publicaciones en Artes y Humanida- des, seguido de Guyana y Haití. Brasil se diferencia de los demás países en cada una de las variables evaluadas (Tabla II).

El objetivo de cualquier editor calificado consiste en posicionar mejor su revista, lo que se logra con un buen apoyo logístico, mediante la participación de profesionales idóneos en la revisión de artículos, en corrección de estilo, en asesoría estadísti$\mathrm{ca}$, que evalúen la idoneidad de los diseños experimentales, técnicas multivariadas, análisis no paramétrico, entre otros métodos. De igual manera, debe existir un buen canal de comunicación entre el editor con las personas que someten un artículo (Espinosa, 2017). Una dificultad muy marcada en el proceso de evaluación se presenta con los evaluadores, pues muchos de ellos no saben manejar adecuadamente las plataformas de las revistas, lo que trae como consecuencia la demora en dar un veredicto definitivo. El par académico se constituye en un juez necesario para garantizar la calidad del escrito (Díaz y otros, 2018).

De acuerdo con los resultados derivados del proceso investigativo, se observa que Brasil es el país de Latinoamérica donde más publicaciones existen en el área de Artes y Humanidades, algo que puede obedecer a que existen más revistas bien categorizadas en el área, lo que hace que los investigadores se motiven, en mayor medida, a someter sus diferentes publicaciones en este país, agregando que muchos medios reciben artículos en inglés, portugués y español, lo que amplía la viabilidad del artículo. Chile posee más publicaciones en los temas de: T4=Conservación, T12=Estudios Religiosos y T13=Arte Visual y Actuación en Arte. En Argentina priman las publicaciones en: T1=Arqueología y T3=Los Clásicos (Tabla IIIa).

Tabla I. Estadísticas promedio anual por tema, en Artes y Humanidades en Latinoamérica

\begin{tabular}{|l|c|c|c|c|c|c|}
\hline TEMA & $\mathbf{V 1}$ & $\mathbf{V 2}$ & $\mathbf{V 3}$ & $\mathbf{V 4}$ & $\mathbf{V 5}$ & V6 \\
\hline T1 & $229 \mathrm{c}$ & $216 \mathrm{c}$ & $1537 \mathrm{c}$ & $628 \mathrm{~b}$ & $8 \mathrm{c}$ & $16 \mathrm{~b}$ \\
\hline T2 & $577 \mathrm{~b}$ & $543 \mathrm{~b}$ & $4212 \mathrm{~b}$ & $878 \mathrm{~b}$ & $16 \mathrm{c}$ & $27 \mathrm{~b}$ \\
\hline T3 & $22 \mathrm{e}$ & $23 \mathrm{e}$ & $9 \mathrm{e}$ & $2 \mathrm{~g}$ & $1 \mathrm{c}$ & $1 \mathrm{c}$ \\
\hline T4 & $74 \mathrm{~d}$ & $67 \mathrm{~d}$ & $114 \mathrm{~d}$ & $32 \mathrm{f}$ & $37 \mathrm{~b}$ & $4 \mathrm{c}$ \\
\hline T5 & $533 \mathrm{~b}$ & $514 \mathrm{~b}$ & $1157 \mathrm{c}$ & $424 \mathrm{c}$ & $2 \mathrm{c}$ & $12 \mathrm{~b}$ \\
\hline T6 & $784 \mathrm{a}$ & $758 \mathrm{a}$ & $1216 \mathrm{c}$ & $519 \mathrm{c}$ & $2 \mathrm{c}$ & $12 \mathrm{~b}$ \\
\hline T7 & $374 \mathrm{c}$ & $337 \mathrm{~b}$ & $14792 \mathrm{a}$ & $1505 \mathrm{a}$ & $73 \mathrm{a}$ & $43 \mathrm{a}$ \\
\hline T8 & $488 \mathrm{~b}$ & $469 \mathrm{~b}$ & $1236 \mathrm{c}$ & $274 \mathrm{~d}$ & $3 \mathrm{c}$ & $13 \mathrm{~b}$ \\
\hline T9 & $417 \mathrm{~b}$ & $384 \mathrm{~b}$ & $198 \mathrm{~d}$ & $85 \mathrm{e}$ & $1 \mathrm{c}$ & $4 \mathrm{c}$ \\
\hline T10 & $94 \mathrm{~d}$ & $93 \mathrm{c}$ & $229 \mathrm{~d}$ & $109 \mathrm{e}$ & $5 \mathrm{c}$ & $5 \mathrm{c}$ \\
\hline T11 & $79 \mathrm{~d}$ & $106 \mathrm{c}$ & $195 \mathrm{~d}$ & $48 \mathrm{f}$ & $1 \mathrm{c}$ & $3 \mathrm{c}$ \\
\hline T12 & $96 \mathrm{~d}$ & $95 \mathrm{c}$ & $88 \mathrm{~d}$ & $28 \mathrm{f}$ & $1 \mathrm{c}$ & $3 \mathrm{c}$ \\
\hline T13 & $192 \mathrm{c}$ & $178 \mathrm{c}$ & $99 \mathrm{~d}$ & $32 \mathrm{f}$ & $1 \mathrm{c}$ & $3 \mathrm{c}$ \\
\hline MANOVA & Wilks' L & Pillai's T & Hotelling & Roy's G & & $\mathbf{p}<\mathbf{0 , 0 0 0 1}$ \\
\hline
\end{tabular}

T1=Arqueología, T2=Artes y Humanidades, T3=Los Clásicos, T4=Conservación, T5= Historia, T6=Historia y Filosofía de la Ciencia, T7=Lenguaje y Lingüística, T8=Literatura y Teoría Literaria, T9=Museología, T10=Música, T11=Filosofía, T12=Estudios Religiosos, T13=Arte Visual y Actuación en Arte. V1=documentos publicados, V2=documentos citados, V3= citaciones, V4=auto citaciones, $\mathrm{V} 5=$ citaciones promedio por documento, V6= índice $\mathrm{H}$. Letras distintas indican diferencia en cada una de las variables. 
Tabla II. Estadísticas en el área de Artes y Humanidades en Latinoamérica (1996-2018)

\begin{tabular}{|c|c|c|c|c|c|c|c|}
\hline País & V1 & $\mathbf{V} 2$ & v3 & V4 & V5 & V6 & Comparación \\
\hline Argentina & 7404 & 7075 & 54169 & 9706 & 7,32 & 90 & $b$ \\
\hline Bolivia & 159 & 153 & 4652 & 295 & 29,26 & 29 & $d$ \\
\hline Brasil & 16143 & 15304 & 129708 & 27405 & 8,03 & 152 & a \\
\hline Chile & 7791 & 7350 & 41229 & 8583 & 5,29 & 84 & b \\
\hline Colombia & 4225 & 3925 & 19702 & 2248 & 4,66 & 57 & $\mathrm{~b}$ \\
\hline Ecuador & 456 & 431 & 6504 & 321 & 14,26 & 31 & $\mathrm{c}$ \\
\hline Paraguay & 32 & 31 & 2259 & 6 & 70,59 & 6 & $\mathrm{e}$ \\
\hline Perú & 789 & 744 & 11780 & 784 & 14,93 & 41 & c \\
\hline Uruguay & 447 & 430 & 6230 & 294 & 13,94 & 26 & c \\
\hline Venezuela & 998 & 942 & 12461 & 798 & 12,49 & 44 & c \\
\hline Costa Rica & 273 & 257 & 7552 & 180 & 27,66 & 23 & d \\
\hline Cuba & 395 & 371 & 3534 & 339 & 8,95 & 27 & c \\
\hline Salvador & 56 & 55 & 152 & 8 & 2,71 & 7 & $\mathrm{e}$ \\
\hline Guatemala & 99 & 98 & 1149 & 80 & 11,61 & 19 & $\mathrm{e}$ \\
\hline Honduras & 24 & 22 & 1197 & 3 & 49,88 & 7 & e \\
\hline México & 6836 & 6496 & 81870 & 8186 & 11,98 & 111 & $\mathrm{~b}$ \\
\hline Nicaragua & 36 & 30 & 450 & 29 & 12,50 & 7 & $\mathrm{e}$ \\
\hline Panamá & 183 & 157 & 18333 & 955 & 100,18 & 53 & $d$ \\
\hline Puerto Rico & 398 & 370 & 9614 & 423 & 24,16 & 43 & $\mathrm{C}$ \\
\hline Rep. Dom. & 59 & 54 & 496 & 47 & 8,41 & 11 & $\mathrm{e}$ \\
\hline Surinam & 8 & 7 & 268 & 1 & 33,50 & 4 & $\mathrm{e}$ \\
\hline Guyana & 12 & 12 & 25 & 0 & 2,04 & 4 & $\mathrm{e}$ \\
\hline Haití & 34 & 34 & 1645 & 30 & 48,38 & 10 & $\mathrm{e}$ \\
\hline Jamaica & 192 & 180 & 1667 & 62 & 8,68 & 17 & $d$ \\
\hline \multicolumn{2}{|c|}{ Wilks' L $p<0,0001$} & \multicolumn{2}{|c|}{ Pillai's T $p<0,0001$} & \multicolumn{2}{|c|}{ Hotelling $p<0,0001$} & \multicolumn{2}{|r|}{$<0,0001$} \\
\hline
\end{tabular}

T1=Arqueología, T2=Artes y Humanidades, T3=Los Clásicos, T4=Conservación, T5= Historia, T6=Historia y Filosofía de la Ciencia, T7=Lenguaje y Lingüística, T8=Literatura y Teoría Literaria, T9=Museología, T10=Música, T11=Filosofía, T12=Estudios Religiosos, T13=Arte Visual y Actuación en Arte.

Tabla IIIa. Países de Latinoamérica con mayor número de publicaciones (1996-2018)

\begin{tabular}{|c|c|c|c|c|c|c|c|c|}
\hline \multirow{2}{*}{$\begin{array}{l}\text { Tema } \\
\text { T1 }\end{array}$} & \multicolumn{2}{|c|}{ Primero } & \multicolumn{2}{|c|}{ Segundo } & \multicolumn{2}{|c|}{ Tercero } & \multicolumn{2}{|c|}{ Cuarto } \\
\hline & Argentina & 1131 & México & 728 & Brasil & 634 & Chile & 448 \\
\hline $\mathrm{T2}$ & Brasil & 2234 & Chile & 1646 & México & 1510 & Colombia & 1123 \\
\hline T3 & Argentina & 130 & Brasil & 95 & Chile & 53 & México & 24 \\
\hline T4 & Chile & 290 & Colombia & 187 & Brasil & 166 & México & 104 \\
\hline T5 & Brasil & 3733 & Argentina & 2038 & México & 1753 & Chile & 1625 \\
\hline T6 & Brasil & 2206 & Argentina & 875 & México & 787 & Chile & 540 \\
\hline T7 & Brasil & 2942 & Chile & 1183 & Argentina & 872 & México & 793 \\
\hline T8 & Brasil & 1930 & Chile & 1685 & Argentina & 829 & México & 650 \\
\hline T9 & Brasil & 734 & Colombia & 192 & México & 51 & Argentina & 50 \\
\hline T10 & Brasil & 677 & Chile & 219 & Colombia & 206 & Argentina & 104 \\
\hline T11 & Brasil & 2717 & Argentina & 1493 & México & 1028 & Chile & 1026 \\
\hline T12 & Chile & 433 & Brasil & 342 & Argentina & 211 & México & 190 \\
\hline T13 & Chile & 852 & Brasil & 517 & México & 401 & Colombia & 399 \\
\hline
\end{tabular}

T1=Arqueología, T2=Artes y Humanidades, T3=Los Clásicos, T4=Conservación, T5= Historia, T6=Historia y Filosofía de la Ciencia, T7=Lenguaje y Lingüística, T8=Literatura y Teoría Literaria, T9=Museología, T10=Música, T11=Filosofía, T12=Estudios Religiosos, T13=Arte Visual y Actuación en Arte. 
El análisis de clúster permitió ubicar a Brasil en el clúster uno, como el país que presenta el mayor número de publicaciones en Artes y Humanidades, diferenciándose de manera notoria respecto a las demás naciones de Latinoamérica. El segundo grupo lo establecen: Chile, Argentina y México. Los países de Centroamérica ocupan los últimos clúster, debido al bajo número de publicaciones (Tabla IIIb).

Los países de Latinoamérica con el mayor número de citaciones en el área de Artes y Humanidades son: Brasil, Argentina, Chile, México y Colombia. El tema con mayor número de citaciones corresponde a: T6=Historia y Filosofía de la Ciencia, y el de menor número corresponde a: T3=Los Clásicos (Tabla IVa).

Al efectuar el análisis de clúster relacionado con el número total de citaciones en Artes y Humanidades en Latinoamérica, Brasil ocupa el primer lugar, seguido de México, Argentina y Chile, anotando que estas naciones ocupan diferentes grupos, como se aprecia en la Tabla IVb.
Respecto al número promedio de documentos citados, Brasil, México, Argentina, Chile y Colombia ocupan las primeras ubicaciones en Latinoamérica. Los temas más referenciados son: T6=Historia y Filosofía de la Ciencia, T2=Artes y Humanidades, T1=Arqueología y T9=Museología (Tabla V).

La preferencia por una revista depende de varios factores. En primera instancia, se establece el perfil de la revista y el idioma en que se publica; el lenguaje ha sido un obstáculo importante para la publicación en revistas emitidas en inglés, aunque esta barrera se está superando por el aumento de investigadores de habla inglesa. En segunda instancia, las universidades dan mayor importancia a los recursos económicos asignados a la investigación, así como algunas medidas adoptadas para facilitar la traducción al idioma inglés. En la actualidad, muchas revistas que se emiten en Colombia, permiten publicar artículos en inglés, y algunas de ellas efectúan la traducción de los manuscritos presentados (Acuña y otros, 2013).

Tabla IIIb. Análisis de clúster, número total de publicaciones en Artes y Humanidades

\begin{tabular}{|c|c|c|c|c|c|c|c|c|c|}
\hline $\begin{array}{l}\text { Brasil } \\
16143\end{array}$ & 1 & $\begin{array}{l}\text { Chile } \\
7791\end{array}$ & 2 & $\begin{array}{l}\text { Argentina } \\
7404\end{array}$ & 2 & $\begin{array}{l}\text { México } \\
6836\end{array}$ & 2 & $\begin{array}{l}\text { Colombia } \\
4225\end{array}$ & 3 \\
\hline $\begin{array}{l}\text { Venezuela } \\
928\end{array}$ & 4 & $\begin{array}{l}\text { Perú } \\
789\end{array}$ & 4 & $\begin{array}{l}\text { Ecuador } \\
456\end{array}$ & 5 & $\begin{array}{l}\text { Uruguay } \\
447\end{array}$ & 5 & $\begin{array}{l}\text { Puerto Rico } \\
398\end{array}$ & 5 \\
\hline $\begin{array}{l}\text { Cuba } \\
395\end{array}$ & 5 & $\begin{array}{l}\text { Costa Rica } \\
273\end{array}$ & 6 & $\begin{array}{l}\text { Jamaica } \\
192\end{array}$ & 6 & $\begin{array}{l}\text { Panamá } \\
183\end{array}$ & 6 & $\begin{array}{l}\text { Bolivia } \\
159\end{array}$ & 6 \\
\hline $\begin{array}{l}\text { Guatemala } \\
99\end{array}$ & 7 & $\begin{array}{l}\text { R. Dominicana } \\
59\end{array}$ & 7 & $\begin{array}{l}\text { El Salvador } \\
56\end{array}$ & 7 & $\begin{array}{l}\text { Nicaragua } \\
36\end{array}$ & 7 & $\begin{array}{l}\text { Haití } \\
34\end{array}$ & 8 \\
\hline $\begin{array}{l}\text { Paraguay } \\
32\end{array}$ & 8 & $\begin{array}{l}\text { Honduras } \\
24\end{array}$ & 8 & $\begin{array}{l}\text { Guyana } \\
12\end{array}$ & 9 & $\begin{array}{l}\text { Suriname } \\
8\end{array}$ & 9 & & \\
\hline MANOVA & & $\begin{array}{c}\text { Wilks' } \\
p<0,0001\end{array}$ & & $\begin{array}{r}\text { Pilla } \\
p<0, C\end{array}$ & & $\begin{array}{l}\text { Hote } \\
p<0,\end{array}$ & & $\begin{array}{r}\text { Roy } \\
p<0,\end{array}$ & \\
\hline
\end{tabular}

Número en negrilla representa el clúster.

Tabla IVa. Países de Latinoamérica con mayor número de citaciones (1996-2018)

\begin{tabular}{|c|c|c|c|c|c|c|c|}
\hline Tema & Primero & \multicolumn{2}{|c|}{ Segundo } & \multicolumn{2}{|c|}{ Tercero } & \multicolumn{2}{|c|}{ Cuarto } \\
\hline T1 & Argentina 7405 & Brasil & 5076 & México & 4185 & Chile & 3511 \\
\hline T2 & 25476 & México & 16143 & Chile & 8627 & Colombia & 5301 \\
\hline T3 & Argentina & Brasil & 28 & Chile & 6 & México & 4 \\
\hline T4 & Brasil & México & 398 & Chile & 168 & Colombia & 137 \\
\hline T5 & Argentina 5496 & Brasil & 5225 & México & 3856 & Chile & 2795 \\
\hline T6 & 77008 & México & 52131 & Argentina & 32212 & Chile & 22871 \\
\hline T7 & Brasil & México & 3720 & Chile & 3320 & Argentina & 2851 \\
\hline T8 & Chile & Brasil & 1032 & Argentina & 851 & México & 825 \\
\hline T9 & Brasil & Argentina & 528 & Colombia & 287 & México & 183 \\
\hline T10 & Brasil & Argentina & 409 & Colombia & 256 & Chile & 147 \\
\hline T11 & Brasil & México & 3495 & Argentina & 2423 & Chile & 1160 \\
\hline T12 & Brasil & Chile & 292 & México & 196 & Argentina & 190 \\
\hline T13 & Brasil & México & 496 & Chile & 287 & Colombia & 193 \\
\hline
\end{tabular}

T1=Arqueología, T2=Artes y Humanidades, T3=Los Clásicos, T4=Conservación, T5= Historia, T6=Historia y Filosofía de la Ciencia, T7=Lenguaje y Lingüística, T8=Literatura y Teoría Literaria, T9=Museología, T10=Música, T11=Filosofía, T12=Estudios Religiosos, T13=Arte Visual y Actuación en Arte. 
Tabla IVb. Análisis clúster, número de citaciones en Artes y Humanidades

\begin{tabular}{|c|c|c|c|c|c|c|c|c|}
\hline $\begin{array}{l}\text { Brasil } \\
129708\end{array}$ & 1 & $\begin{array}{l}\text { México } \\
81870\end{array}$ & 2 & $\begin{array}{l}\text { Argentina } \\
54162\end{array}$ & 3 & $\begin{array}{l}\text { Chile } \\
41229\end{array}$ & 4 & $\begin{array}{l}\text { Colombia } \\
19702\end{array}$ \\
\hline $\begin{array}{l}\text { Panamá } \\
18333\end{array}$ & 5 & $\begin{array}{l}\text { Venezuela } \\
\mathbf{6}\end{array}$ & 12461 & $\begin{array}{l}\text { Perú } \\
11780\end{array}$ & 6 & $\begin{array}{l}\text { Puerto ricc } \\
9614\end{array}$ & 6 & $\begin{array}{l}\text { Costa Rica } \\
7552\end{array}$ \\
\hline $\begin{array}{l}\text { Ecuador } \\
6504\end{array}$ & 7 & $\begin{array}{l}\text { Uruguay } \\
6230\end{array}$ & 7 & $\begin{array}{l}\text { Bolivia } \\
4652\end{array}$ & 8 & $\begin{array}{l}\text { Cuba } \\
3534\end{array}$ & 8 & $\begin{array}{l}\text { Paraguay } \\
2259\end{array}$ \\
\hline $\begin{array}{l}\text { Jamaica } \\
1667\end{array}$ & 9 & $\begin{array}{l}\text { Haití } \\
1645\end{array}$ & 9 & $\begin{array}{l}\text { Honduras } \\
1197\end{array}$ & 9 & $\begin{array}{l}\text { Guatemala } \\
1149\end{array}$ & 9 & $\begin{array}{l}\text { R. Dominicana } \\
496 \quad 10\end{array}$ \\
\hline $\begin{array}{l}\text { Nicaragua } \\
450\end{array}$ & 10 & $\begin{array}{l}\text { Suriname } \\
268\end{array}$ & 10 & $\begin{array}{l}\text { El salvador } \\
152\end{array}$ & 10 & $\begin{array}{l}\text { Guyana } \\
25\end{array}$ & 11 & \\
\hline \multicolumn{2}{|c|}{ MANOVA } & \multicolumn{2}{|c|}{$\begin{array}{c}\text { Wilks' } \\
p<0,0001\end{array}$} & \multicolumn{2}{|c|}{$\begin{array}{c}\text { Pillai's } \\
p<0,0001\end{array}$} & \multicolumn{2}{|c|}{$\begin{array}{l}\text { Hotelling } \\
p<0,0001\end{array}$} & $\begin{array}{c}\text { Roy's G } \\
p<0,0001\end{array}$ \\
\hline
\end{tabular}

Número en negrilla representa el clúster.

Tabla V. Promedio de citaciones en Artes y Humanidades en Latinoamérica (1996-2018)

\begin{tabular}{|c|c|c|c|c|c|c|c|c|}
\hline \multirow{2}{*}{$\begin{array}{l}\text { Tema } \\
\text { T1 } \\
\end{array}$} & \multicolumn{2}{|c|}{ Primero } & \multicolumn{2}{|c|}{ Segundo } & \multicolumn{2}{|c|}{ Tercero } & \multicolumn{2}{|c|}{ Cuarto } \\
\hline & Brasil & 8,01 & Chile & 7,84 & Argentina & 6,55 & México & 5,75 \\
\hline T2 & Brasil & 11,40 & México & 10,69 & Chile & 5,24 & Colombia & 4,72 \\
\hline T3 & Argentina & 0,52 & Brasil & 0,29 & México & 0,25 & Chile & 0,21 \\
\hline T4 & México & 3,85 & Brasil & 3,55 & Colombia & 0,73 & Chile & 0,58 \\
\hline T5 & Argentina & 2,70 & México & 2,20 & Chile & 1,72 & Brasil & 1,40 \\
\hline T6 & México & 66,24 & Chile & 42,25 & Argentina & 36,81 & Brasil & 34,91 \\
\hline T7 & México & 4,69 & Argentina & 3,27 & Chile & 2,81 & Brasil & 2,36 \\
\hline$T 8$ & México & 1,27 & Argentina & 1,03 & Chile & 0,86 & Brasil & 0,53 \\
\hline T9 & Argentina & 11,96 & México & 3,59 & Brasil & 1,82 & Colombia & 1,49 \\
\hline T10 & Argentina & 3,93 & Brasil & 2,38 & Colombia & 1,24 & Chile & 0,67 \\
\hline T11 & México & 3,40 & Brasil & 3,27 & Argentina & 1,62 & Chile & 1,13 \\
\hline T12 & Brasil & 1,71 & México & 1,03 & Argentina & 0,90 & Chile & 0,67 \\
\hline T13 & Brasil & 1,11 & México & 1,06 & Colombia & 0,48 & Chile & 0,34 \\
\hline MANOVA & $\begin{array}{r}\text { Will } \\
p<0,0\end{array}$ & $\begin{array}{l}\mathbf{r s}^{\prime} \\
001 \\
\end{array}$ & $\begin{array}{r}\text { Pilla } \\
p<0,0\end{array}$ & $\begin{array}{l}\text { i's } \\
001\end{array}$ & Hotelling $p$ & $<0,0001$ & $\begin{array}{r}\text { Roy' } \\
p<0,0\end{array}$ & $\begin{array}{l}\mathbf{G} \\
001\end{array}$ \\
\hline
\end{tabular}

T1=Arqueología, T2=Artes y Humanidades, T3=Los Clásicos, T4=Conservación, T5= Historia, T6=Historia y Filosofía de la Ciencia, T7=Lenguaje y Lingüística, T8=Literatura y Teoría Literaria, T9=Museología, T10=Música, T11=Filosofía, T12=Estudios Religiosos, T13=Arte Visual y Actuación en Arte.

En el presente, se estima que un alto porcentaje de las revistas de alto impacto científico se publican en idioma inglés. En Sudamérica la cifra es muy baja, aproximadamente un 3\%, aunque vale resaltar que esta cifra tiende a incrementar en un futuro cercano debido a la internacionalización del conocimiento, donde gran parte de las revistas son de acceso abierto, lo que facilita la interacción entre investigadores, con la gran ventaja de que puede contribuir a la discusión de los resultados generados en el proceso investigativo. De manera adicional, se puede contactar con los diferentes grupos investigativos o con una persona en particular. También sirve para que los docentes incluyan los resultados más impactantes en sus cátedras. En Latinoamérica, el número de revistas y volúmenes de ellas ha aumentado de ma- nera notoria, debido al avance del internet, que ha permitido ubicar cada medio de difusión científica en la plataforma, con la gran ventaja de que cualquier persona puede acceder a ella de manera gratuita. El problema de la región consiste en que existe un reducido número de investigadores, y pocos de ellos publican. De otro lado, el PIB dedicado a la investigación y al desarrollo tecnológico es precario, como es el caso de Colombia (Aguado y otros, 2015).

Hoy, existen diferentes entes que evalúan la calidad de los artículos científicos publicados, dentro de los que se destacan: SCImago Journal Rank (SJR), Source-Normalized Impact per Paper (SNIP), Factor de Impacto (FI), Arts \& Humanities Citation Index (A\&HCI, 2016), entre otros. En Colombia, Colcien- 
cias (2016) es el ente que clasifica las revistas de forma anual, ubicándolas en el directorio Publindex, en las categorías $A 1, A 2, B$ y $C$, de acuerdo con la calidad del medio de difusión científica. La tendencia de muchas revistas publicadas en Sudamérica es emitir los artículos en inglés, o en forma bilingüe, de manera simultánea, debido a la internacionalización del conocimiento, donde el idioma inglés se declara como lengua universal en el área científica. En la Tabla VI se destaca, para cada tema, el análisis de clúster, el cual permitió clasificar a los diferentes países de Latinoamérica en el área de Artes y Humanidades. El clúster tres corresponde a los países con mayor número de publicaciones y citaciones, donde Brasil domina en todas las clasificaciones. La mayoría de revistas de ese país son de alto nivel académico y se publican en inglés, y le sigue Chile, Argentina y México. Los países de Centroamérica corresponden al clúster de menor productividad.

El análisis de componentes principales permitió establecer tres factores: el primer factor se asoció con los temas: T5 $=$ Historia, T6=Historia y Filosofía de la Ciencia, T7=Lenguaje y Lingüística, T8=Literatura y Teoría Literaria, T10=Música, T11=Filosofía; este factor explica el $78 \%$ de la variabilidad total. El factor dos se correlacionó con: T2=Artes y Humanidades, T4=Conservación, T9=Museología y T12=Estudios Religiosos. El factor tres se asoció con: T1=Arqueología y T3=Los Clásicos, como se aprecia en la Tabla VII.

Tabla VI. Análisis de clúster por tema

\begin{tabular}{|c|c|c|c|c|}
\hline TEMA & Clúster 1 & Clúster 2 & Clúster 3 & Clúster 4 \\
\hline T1 & $\begin{array}{l}\text { Paraguay, Bolivia, } \\
\text { Uruguay, Ecuador, } \\
\text { Venezuela, Panamá }\end{array}$ & $\begin{array}{l}\text { Colombia, Perú, Costa } \\
\text { Rica, Cuba }\end{array}$ & $\begin{array}{l}\text { Chile, Argentina, Brasil, } \\
\text { México }\end{array}$ & $\begin{array}{l}\text { Salvador, Guatemala, } \\
\text { Honduras, Nicaragua }\end{array}$ \\
\hline $\mathbf{T 2}$ & Paraguay, Panamá & $\begin{array}{l}\text { Ecuador, Uruguay, } \\
\text { Venezuela, Bolivia, Perú, } \\
\text { Costa Rica }\end{array}$ & $\begin{array}{l}\text { Brasil, Chile, Argentina, } \\
\text { Colombia, Cuba, } \\
\text { México }\end{array}$ & $\begin{array}{l}\text { Salvador, Guatemala, } \\
\text { Honduras, Nicaragua }\end{array}$ \\
\hline T3 & $\begin{array}{l}\text { Paraguay, Ecuador, Perú, } \\
\text { Uruguay, Venezuela }\end{array}$ & Colombia, Costa Rica & $\begin{array}{l}\text { Brasil, Chile, Argentina, } \\
\text { México, Cuba }\end{array}$ & $\begin{array}{l}\text { Salvador, Guatemala, } \\
\text { Honduras, Nicaragua }\end{array}$ \\
\hline T4 & Paraguay, Panamá & $\begin{array}{l}\text { Bolivia, Perú, Ecuador, } \\
\text { Uruguay, México, Costa Rica }\end{array}$ & $\begin{array}{l}\text { Argentina, Bolivia, Perú, } \\
\text { Ecuador, Brasil, Cuba }\end{array}$ & $\begin{array}{l}\text { Salvador, Guatemala, } \\
\text { Honduras, Nicaragua }\end{array}$ \\
\hline T5 & Paraguay, Panamá & $\begin{array}{l}\text { Bolivia, Ecuador, Perú, } \\
\text { Venezuela, Costa Rica }\end{array}$ & $\begin{array}{l}\text { Brasil, Colombia, Chile, } \\
\text { México Argentina, } \\
\text { Uruguay }\end{array}$ & $\begin{array}{l}\text { Salvador, Guatemala, } \\
\text { Honduras, Nicaragua }\end{array}$ \\
\hline T6 & Paraguay, Panamá & $\begin{array}{l}\text { Perú, Uruguay, } \\
\text { Venezuela, Bolivia, } \\
\text { Ecuador, Costa Rica }\end{array}$ & $\begin{array}{l}\text { Argentina, Brasil, Chile, } \\
\text { Colombia, México }\end{array}$ & $\begin{array}{l}\text { Salvador, Guatemala, } \\
\text { Honduras, Nicaragua }\end{array}$ \\
\hline T7 & $\begin{array}{l}\text { Paraguay, Bolivia, } \\
\text { Panamá }\end{array}$ & $\begin{array}{l}\text { Ecuador, Uruguay, } \\
\text { Colombia, Perú, } \\
\text { Venezuela }\end{array}$ & $\begin{array}{l}\text { Brasil, Chile, Argentina, } \\
\text { México, Costa Rica }\end{array}$ & $\begin{array}{l}\text { Salvador, Guatemala, } \\
\text { Honduras, Nicaragua }\end{array}$ \\
\hline T8 & Paraguay, Bolivia & $\begin{array}{l}\text { Ecuador, Uruguay, Perú, } \\
\text { Costa Rica }\end{array}$ & $\begin{array}{l}\text { Colombia, Brasil, Vene- } \\
\text { zuela, Argentina, Chile, } \\
\text { México }\end{array}$ & $\begin{array}{l}\text { Salvador, Guatemala, } \\
\text { Honduras, Nicaragua }\end{array}$ \\
\hline T9 & Paraguay, Panamá & $\begin{array}{l}\text { Bolivia, Ecuador, Uru- } \\
\text { guay, Perú, Venezuela, } \\
\text { Costa Rica }\end{array}$ & $\begin{array}{l}\text { Brasil, Chile, Argentina, } \\
\text { Colombia, México }\end{array}$ & $\begin{array}{l}\text { Salvador, Guatemala, } \\
\text { Honduras, Nicaragua }\end{array}$ \\
\hline T10 & Paraguay, Panamá & $\begin{array}{l}\text { Chile, Perú, Venezuela, } \\
\text { Uruguay, Bolivia, Ecuador }\end{array}$ & $\begin{array}{l}\text { Argentina, Brasil, } \\
\text { México, Costa Rica }\end{array}$ & $\begin{array}{l}\text { Salvador, Guatemala, } \\
\text { Honduras, Nicaragua }\end{array}$ \\
\hline T11 & $\begin{array}{l}\text { Paraguay, Bolivia, } \\
\text { Panamá }\end{array}$ & $\begin{array}{l}\text { Venezuela, Perú, } \\
\text { Uruguay, Ecuador, Costa } \\
\text { Rica }\end{array}$ & $\begin{array}{l}\text { Brasil, Chile, Colombia, } \\
\text { Argentina, México }\end{array}$ & $\begin{array}{l}\text { Salvador, Guatemala, } \\
\text { Honduras, Nicaragua }\end{array}$ \\
\hline T12 & Paraguay, Panamá & $\begin{array}{l}\text { Bolivia, Perú, Venezuela, } \\
\text { Ecuador, Uruguay }\end{array}$ & $\begin{array}{l}\text { Colombia, Argentina, } \\
\text { Brasil, Chile, México }\end{array}$ & $\begin{array}{l}\text { Salvador, Guatemala, } \\
\text { Honduras, Nicaragua }\end{array}$ \\
\hline T13 & Paraguay, Panamá & $\begin{array}{l}\text { Venezuela, Bolivia, Perú, } \\
\text { Ecuador, Uruguay }\end{array}$ & $\begin{array}{l}\text { Colombia, Brasil, } \\
\text { Argentina, Chile, } \\
\text { México, Costa Rica } \\
\end{array}$ & $\begin{array}{l}\text { Salvador, Guatemala, } \\
\text { Honduras, Nicaragua }\end{array}$ \\
\hline
\end{tabular}

T1=Arqueología, T2=Artes y Humanidades, T3=Los Clásicos, T4=Conservación, T5= Historia, T6=Historia y Filosofía de la Ciencia, T7=Lenguaje y Lingüística, T8=Literatura y Teoría Literaria, T9=Museología, T10=Música, T11=Filosofía, T12=Estudios Religiosos, T13=Arte Visual y Actuación en Arte. 
Tabla VII. Análisis de componentes principales

\begin{tabular}{|l|c|c|c|}
\hline Tema & Factor $\mathbf{1}$ & Factor 2 & Factor 3 \\
\hline T1 & 0,26428 & 0,30853 & 0,92465 \\
\hline T2 & 0,65746 & 0,69253 & 0,38079 \\
\hline T3 & 0,26521 & 0,25241 & 0,94734 \\
\hline T4 & 0,26523 & 0,95461 & 0,09576 \\
\hline T5 & 0,78644 & 0,38015 & 0,50854 \\
\hline T6 & 0,76505 & 0,46678 & 0,48078 \\
\hline T7 & 0,85782 & 0,25349 & 0,46357 \\
\hline T8 & 0,84323 & 0,42458 & 0,34844 \\
\hline T9 & 0,54326 & 0,75279 & 0,38388 \\
\hline T10 & 0,98025 & 0,14673 & 0,12436 \\
\hline T11 & 0,82677 & 0,45948 & 0,14827 \\
\hline T12 & 0,29925 & 0,86839 & 0,42974 \\
\hline T13 & 0,25520 & 0,94035 & 0,27645 \\
\hline $\begin{array}{l}\text { Porcentaje acumulado de } \\
\text { variabilidad }\end{array}$ & 0,7945 & 0,9012 & 0,9806 \\
\hline
\end{tabular}

T1=Arqueología, T2=Artes y Humanidades, T3=Los Clásicos, T4=Conservación, T5= Historia, T6=Historia y Filosofía de la Ciencia, T7=Lenguaje y Lingüística, T8=Literatura y Teoría Literaria, T9=Museología, T10=Música, T11=Filosofía, T12=Estudios Religiosos, T13=Arte Visual y Actuación en Arte.

Chile, Colombia, Argentina, México y Brasil, son los países de Latinoamérica que tienen el mayor número de revistas clasificadas en la categoría Q1, que corresponde a las revistas de mejor impacto científico, destacándose el área de Historia y Literatura, con la mayor ponderación (Tabla VIII).

En la clasificación mundial de revistas de carácter científico, Estados Unidos está presente en primer lugar en todas las áreas de Artes y Humanidades. Brasil, Chile y Argentina se destacan en Latinoamérica. A nivel del mundo, ocupan en general posiciones por encima de la casilla diez (Tabla IX).

\section{CONCLUSIONES}

Las revistas científicas son de gran interés para quienes laboran en investigación, ya que posibilitan la difusión de los resultados generados a la luz de un proceso metodológico. En Sudamérica, pocas revistas están clasificadas en alto rango, por lo tanto, los pocos investigadores científicos que existen en la región suelen someter sus artículos a revistas de Norteamérica o Europa, limitando que los medios de difusión científica con que cuenta la región se puedan ubicar en niveles más altos de citaciones.

Tabla VIII. Revistas en Artes y Humanidades clasificadas en Q1

\begin{tabular}{|l|l|l|c|c|}
\hline País & Revista & Área Temática & Índice H & Citas \\
\hline Argentina & Estudios Atacameños & Arqueología & 14 & 66 \\
\hline Argentina & Antiguo Oriente & Clásicos & 3 & 4 \\
\hline Chile & Diálogo Andino & Historia & 3 & 27 \\
\hline Chile & Estudio Histórico Jurídico & Historia & 5 & 13 \\
\hline Colombia & Estudios Sociales & Historia & 8 & 43 \\
\hline Colombia & Análisis Político & Historia & 5 & 20 \\
\hline México & América Latina Historia & Historia & 4 & 18 \\
\hline Chile & Izquierdas & Historia & 3 & 39 \\
\hline Argentina & Páginas & Historia & 1 & 1 \\
\hline Chile & Literatura y Lingüística & Literatura & 6 & 24 \\
\hline Chile & Signos & Literatura & 11 & 34 \\
\hline Chile & Boletín Filología & Literatura & 3 & 14 \\
\hline Chile & Chilena de Literatura & Literatura & 7 & 26 \\
\hline Brasil & Ilha do desterro & Literatura & 4 & 26 \\
\hline Chile & Boletín del Museo de Arte & Artes Visuales & 3 & 16 \\
\hline Colombia & Kepes & Artes Visuales & 2 & 12 \\
\hline
\end{tabular}


Tabla IX. Clasificación mundial de los países en publicaciones en Artes y Humanidades

\begin{tabular}{|c|c|c|c|c|c|}
\hline Área Temática & Primer Puesto & Índice H & $\begin{array}{c}\text { Primer País } \\
\text { Latinoamericano }\end{array}$ & Puesto & Índice $\mathrm{H}$ \\
\hline Arqueología & Estados Unidos & 158 & Argentina & 22 & 36 \\
\hline Artes y Humanidades & Estados Unidos & 422 & Brasil & 29 & 68 \\
\hline Clásicos & Estados Unidos & 34 & Argentina & 23 & 4 \\
\hline Conservación & Estados Unidos & 36 & Chile & 17 & 6 \\
\hline Historia & Estados Unidos & 174 & Brasil & 14 & 27 \\
\hline Filosofía & Estados Unidos & 1002 & Brasil & 13 & 134 \\
\hline Lenguaje y Literatura & Estados Unidos & 272 & Brasil & 16 & 33 \\
\hline Literatura & Estados Unidos & 79 & Brasil & 15 & 13 \\
\hline Museología & Estados Unidos & 74 & Brasil & 3 & 16 \\
\hline Música & Estados Unidos & 135 & Brasil & 9 & 17 \\
\hline Estudios Religiosos & Estados Unidos & 79 & Chile & 29 & 6 \\
\hline Arte Visual & Estados Unidos & 91 & Chile & 16 & 7 \\
\hline
\end{tabular}

Las fuentes de recursos que otorgan las universidades para el apoyo en publicación, son bajos, siendo este un factor limitante para que un grupo científico de a conocer sus resultados en revistas de alto impacto. De manera adicional, se observa que una gran parte de revistas científicas cobran altas sumas de dinero para publicar en ellas, estableciéndose de este modo una competencia de mercado. A ello se suma que en los proyectos aprobados por entes gubernamentales 0 del sector privado, poco se apoya al análisis de la información y mucho menos la publicación del artículo.

La mayoría de revistas de Artes y Humanidades en Sudamérica, tienen pocos números en el año, máximo dos, lo que implica que someter un artículo a estos medios de difusión científica, hace que la espera para la publicación llegue a tardar más de un año, y en muchos casos la información pierde actualidad, debido a los avances científicos.

Las publicaciones en Artes y Humanidades son más complejas en la recopilación y análisis de la información, por lo que se requiere más tiempo para publicar los resultados derivados de los procesos investigativos (Toro, 2017).

\section{REFERENCIAS}

Acuña, E.; Espinosa, M.; Cancino, J. (2013). Ranking de productividad basado en artículos científicos de instituciones forestales chilenas. Bosque 34(2), 211-219. https://doi.org/10.4067/S0717-92002013000200010.

Aguado, E.; Fischman, G.; Alperin, J.; Cetto, A.; Gamboa, J.; Packer, A.; Bongiovani, P.; Gómez, N. ; Oliveira, K.; Degani, F.; da Silva, N.; Marafon, G.; Marin, A; Petralia, S.; Stubrin, L. (2015). Hecho en Latinoamérica: acceso abierto, revistas académicas e innovaciones
Estados Unidos y el Reino Unido son los países que poseen el mayor porcentaje de publicaciones en el área de Artes y Humanidades. Latinoamérica cuenta con un bajo porcentaje (Pimentel y otros, 2016), siendo Brasil el primer país de Suramérica, ocupando la posición 18 a nivel orbital, seguido de Chile, que se ubica en el puesto 34.

Se debería fomentar redes de investigadores a nivel de Latinoamérica en Artes y Humanidades que propicien mayor desarrollo científico, incrementen el número de publicaciones de alta calidad, e incidan de manera significativa en las estadísticas referidas al número de documentos citados e índices de impacto.

\section{AGRADECIMIENTOS}

A la Universidad de Antioquia, por brindarme el tiempo para investigar.

\section{ACKNOLEDGEMENTS}

To the University of Antioquia, for giving me the time to investigate.

regionales ( $1^{\text {a }}$ ed.). Ciudad Autónoma de Buenos Aires: CLACSO. http://biblioteca.clacso.edu.ar/clacso/ se/20150722110704/HechoEnLatinoamerica.pdf [Fecha de consulta: 26/04/2019].

Alcaín, M.; Román, A.; Giménez, E. (2008). Categorización de las revistas españolas de Ciencias Sociales y Humanas en RESH. Revista Española de Documentación Científica, 31 (1), 85-95. http://redc.revistas.csic.es/index.php/ redc/article/view/414 [Fecha de consulta: 14/09/2019]. 
Andronic, O.; Ion, D.; Dumitru, C.; Badiu, A.; Titus, V.; Radu, G.; Păduraru, D.; Bolocan, A. (2017). The evolution of scientific publishing during the last decade. Proc. Rom. Acad., Series B, 19(3), 151-155. https:// www.researchgate.net/publication/322163561 [Fecha de consulta: 01/03/2019].

A\&HCI - Arts \& Humanities Citation Index (via the Web of Science). (2016). Clarivate Analytics. [En línea] Disponible en: http://library.maastrichtuniversity.nl/database/arts/ [Fecha de consulta: 15/11/2019].

Beltrán, O. (2006). Factor de Impacto. Revista Colombiana de Gastroenterología, 21 (1). http://www.scielo. org.co/pdf/rcg/v21n1/v21n1a09.pdf [Fecha de consulta: $13 / 03 / 2019]$

Benavent, A.; Valderrama, J. C.; González, G. (2007). "El factor de impacto de las revistas científicas: limitaciones e indicadores alternativos". El profesional de la información, 16 (1), 4-11. http://eprints.rclis. org/9489/. [Fecha de consulta: 05/03/2019].

Bienert, I.R.; Carvalho, R.; de Andrade, P.A.; Caramori, C. (2015). Bibliometric indexes, databases and impact factors in cardiology. Braz J Cardiovasc Surg, 30 (2), 254259. https://doi.org/10.5935/1678-9741.20150019

Buela, G. (2003). Evaluación de la calidad de los artículos y de las revistas científicas: Propuesta del factor de impacto ponderado y de un índice de calidad. Psicothema, 15 (1), 23-35. http://www.psicothema.com/psicothema.asp?id=400 [Fecha de consulta: 10/03/2019].

Caerols, R.; Verdú, S.; Viñarás, M. (2017). Las artes en la educación superior: la evaluación en España de la actividad investigadora en Bellas Artes a través de los indicadores de calidad. Revista Española de Documentación Científica, 40 (4), e191. http://dx.doi. org/10.3989/redc.2017.4.1394

Colciencias. (2016). Política nacional para mejorar el impacto de las publicaciones científicas nacionales. https://minciencias.gov.co/sites/default/files/upload/ noticias/120816-vfpolitica_publindex_2.0_og_ao_miv. pdf [Fecha de consulta: 20/04/2019].

Delgado, E.; Ruiz, R.; Jiménez, E. (2006). La edición de revistas científicas. Directrices, criterios y modelos de evaluación. Universidad de Granada. Grupo de Investigación "EC3: Evaluación de la Ciencia y de la Comunicación Científica". https://www.fecyt.es/es/publicacion/la-edicion-de-revistas-cientificas-directricescriterios-y-modelos-de-evaluacion. [Fecha de consulta: $14 / 02 / 2019]$

Díaz, G.; Hernández, V.; Núñez, I. (2018). Convergencia Revista de Ciencias Sociales y los avatares hacia su digitalización. En: Rus, J.; Pinto, A. M. (coords.), Revistas científicas mexicanas: Retos de calidad y visibilidad en acceso abierto, pp. 15-22. Universidad de Ciencias y Ar- tes de Chiapas Centro de Estudios Superiores de México y Centroamérica. http://209.177.156.169/libreria_cm/ archivos/pdf_1673.pdf. [Fecha de consulta: 26/02/2019].

Espinosa, F. (2017). La calidad de una publicación científica es resultado de esfuerzos y atributos. Gaceta Médica de México, 153, 293-296. Editorial. https://www.anmm. org.mx/GMM/2017/n3/GMM_153_2017_3_293-296. pdf. [Fecha de consulta: 21/03/2019].

Jiménez, A. (2015). Tipos de publicaciones científicas. Orthotips. 11 (2), 58-67. https://www.medigraphic.com/ pdfs/orthotips/ot-2015/ot152b.pdf [Fecha de consulta: 22/04/2019]

López, E. (2016). La importancia de las publicaciones científicas. Actualización en Nutrición, 17 (1): 1-2. http://www.revistasan.org.ar/pdf files/trabajos/ vol_17/num_1/RSAN_17_1_1.pdf. [Fecha de consulta: $15 / 04 / 2019]$

Pimentel, G.; Garcés, A.; Kuzminsky, S. C.; Agüero, C.; Núñez, L. (2016). El conocimiento en disputa: Algunas observaciones sobre cienciometría, sistemas de acceso y ciencia social latinoamericana. Estudios atacameños, 53, 3-9. https://doi.org/10.4067/S071810432016000200001

Romanos, S. (2008). La revista científica: Panorama internacional, latinoamericano y argentino. En: Romanos, S., Revistas Argentinas de Humanidades y Ciencias Sociales. Visibilidad en Bases de datos internacionales. Buenos Aires: UBA.

Rozemblum, C.; Unzurrunzaga, C.; Pucacco, C.; Banzato, G. (2012). Parámetros de evaluación para la inclusión e indización de revistas científicas en bases de datos locales e internacionales. Análisis sobre su aporte a la calidad de las publicaciones de Humanidades y Ciencias Sociales. VII Jornadas de Sociología de la UNLP. Argentina en el escenario latinoamericano actual: debates desde las ciencias sociales, 5 al 7 de diciembre de 2012, La Plata. Disponible en: http://www.memoria.fahce.unlp.edu.ar/trab_eventos/ev.1406/ev.1406. pdf [Fecha de consulta: [23/02/2019].

Testa, J. (2009). Proceso de selección de revistas especializadas de Thompson Reuters. https://biblioteca.uah. es/biblioteca/documentos/journal_selection_essay-es. pdf [Fecha de consulta: 01/05/2019].

Toro, I. (2017). La bibliometría y las publicaciones en teología. Cuest. teol. 44 (102), 229-239. https://doi. org/10.18566/cueteo.v44n102.a01

Torres, D.; Bordons, M.; Giménez, E.; Delgado, C.; Jiménez, E.; Sanz, E. (2010). Clasificación integrada de revistas científicas (CIRC): propuesta de categorización de las revistas en ciencias sociales y humanas. El profesional de la información, 19 (6). https://doi. org/10.3145/epi.2010.nov.15 